\title{
Analysis of the amplitude changes and baseline shifts of respiratory motion using intra-fractional CBCT in liver stereotactic body radiation therapy
}

\author{
Lu Zeng ${ }^{\mathrm{a}}$, Xin Wang ${ }^{\mathrm{a}}$, Jidan Zhou ${ }^{\mathrm{a}}$, Pan Gong ${ }^{\mathrm{a}}$, Xuetao Wang ${ }^{\mathrm{a}}$, Xiaohong Wu ${ }^{\mathrm{b}}$, \\ Zhonghua Deng ${ }^{a}$, Bin $\mathrm{Li}^{\mathrm{a}}$, Denghong Liu ${ }^{\mathrm{a}}$, Renming Zhong ${ }^{\mathrm{a}}$, \\ ${ }^{a}$ Department of Radiotherapy, State Key Laboratory of Biotherapy and Cancer Center, West China Hospital, Sichuan University, Chengdu 610041, PR China \\ ${ }^{\mathrm{b}}$ Department of Oncology, The Affiliated Hospital of Panzhihua University, Panzhihua 617099, PR China
}

\section{A R T I C L E I N F O}

\section{Keywords:}

4D CT

Intra-fractional CBCT

Liver SBRT

Amplitude changes and baseline shifts

ITV to PTV margin

\begin{abstract}
A B S T R A C T
Purpose: Using intra-fractional cone-beam CT (CBCT) to evaluate the amplitude changes and baseline shifts of respiratory motion in liver stereotactic body radiation therapy (SBRT).

Methods: The amplitude changes and baseline shifts of respiratory motion for 24 liver patients were evaluated by the four-dimensional (4D) CT, inter- and intra-fractional CBCT. The difference of the average liver position errors among all treatment fractions and the 4D CT representthe baseline shifts. According to the baseline shifts, the ITV to PTV margin was recalculated and the plan was re-designed to compare the dosimetric variation.

Results: The systematic and random errors of the baseline shifts for intra-fractional CBCT in the left-right (LR), superior-inferior (SI), and anterior-posterior (AP) directions were 0.99/1.60 mm, 2.03/2.46 mm, and 1.02/2.07 $\mathrm{mm}$, respectively. The new ITV to PTV margins should be $4.0 \mathrm{~mm}, 7.0 \mathrm{~mm}$, and $4.0 \mathrm{~mm}$, respectively. The amplitude change of motion between the 4D CT and the intra-fractional CBCT was $1.03 \pm 4.35 \mathrm{~mm}$, with $31 \%$ of fractions exceeding $5 \mathrm{~mm}$. To achieve the same dose coverage of the new PTV, the $\mathrm{D}_{\text {mean }}, \mathrm{V} 50, \mathrm{~V} 40, \mathrm{~V} 30, \mathrm{~V} 25$ of normal liver and maximum dose of the duodenum were significantly different.

Conclusions: Significant amplitude changes and baseline shifts of motion occurred during dose delivery compared with those in 4D CT. Using the ITV to PTV margin of $4.0 \mathrm{~mm}$ (LR), $7.0 \mathrm{~mm}$ (SI), and $4.0 \mathrm{~mm}$ (AP) can ensure the target dose coverage and keep the dose constrain of normal tissues at an acceptable level.
\end{abstract}

\section{Background}

Stereotactic body radiation therapy (SBRT) has become one of the main treatments for inoperable liver cancer and liver metastases and can significantly improve the local control rate of cancer in patients. In SBRT, steep dose gradients exist around the target volume, and therefore a tiny position deviation can cause a significant change to the target dose. The localization of the target area of the liver is affected by interfractional errors (depends on different immobilization devices and setup errors) and respiratory motion [1]. Therefore, image-guided radiotherapy (IGRT) and respiratory motion management strategies are necessary to correct these errors and improve the accuracy of radiotherapy [2]. The IGRT strategies include cone-beam CT (CBCT), kilovoltage (kV) planar imaging [3], magnetic resonance (MR) [4] and electronic portal imaging device (EPID), etc. Respiratory motion management includes expanded margin under free-breathing, 4D CT [5], tracking [6], gating, and deep inspiration breath-hold (DIBH) [1]. CBCTguided DIBH technology can reduce the patient's respiration motion; however, some patients cannot tolerate this DIBH technology well, and a more considerable ITV to PTV margin is needed to compensate for the variation between different breath-holds [5]. Gating and tracking technologies usually require additional auxiliary equipment and implanted benchmarks. Therefore, 4D CT is still the most popular method for respiratory motion management for liver SBRT under freebreathing.

In clinical practice, the 4D CT is used to generate an internal target volume (ITV) [7] by either combining the targets contoured on each respiratory phase (ten phases in total) or using a maximum intensity project (MIP) [8] image; alternatively, the mid-ventilation CT can be used to delineate the target [9]. There is an assumption that the respiratory motion had no long-term changes in following treatment fractions (especially when dose delivery), such as baseline drifts or amplitude

\footnotetext{
* Corresponding author at: Department of Radiotherapy, Division of Radiation Physics, State Key Laboratory of Biotherapy and Cancer Center, West China Hospital, Sichuan University, Chengdu 610041, PR China..

E-mail address: zrm_100@163.com (R. Zhong).
} 
variation. The baseline shifts were defined as the difference of the average tumor position (or average liver position) among all treatment fractions and the 4D CT [10]. So the $5 \mathrm{~mm}$ uniform ITV to PTV margins have been widely used in liver SBRT patients who underwent 4D CT with free-breathing.

Up to now, to analyse intra-fractional errors, many approaches have been proposed, including post-treatment CBCT [11-13], KV fluoroscopy $[6,14]$, and electromagnetic tracking [15]. The KV fluoroscopy is a form of two-dimensional (2D) imaging, similar to electromagnetic tracking, lacking soft tissue information, which usually requires the implantation of metal markers as a surrogate to determine the target localization. Other studies reported that they evaluated the intra-fractional errors by using post-treatment CBCT. However, that may not be a sufficient surrogate for the errors during dose delivery.

The intra-fractional CBCT was acquired during the dose delivery, representing the $3 \mathrm{D}$ intra-fractional errors in real-time. Li et al. [16] used intra-fractional CBCT images to assess intra-fractional geometric and dosimetric accuracy of stereotactic ablative radiation therapy and confirmed large intra-fractional errors in the lung SBRT. Poulsen P R et al. [17] used intra-fractional kilovoltage (2D kV image) imaging to estimate the intra-treatment liver target motion. However, no study has investigated intra-fractional errors for liver SBRT with free-breathing using intra-fractional СВСТ.

In this study, we analyzed the amplitude changes and baseline shifts of respiratory motion during liver SBRT dose delivery, using inter- and intra-fractional CBCT. Due to the inter-fractional errors eliminated by pre-treatment image guidance, the ITV to PTV margin was used to compensate for intra-fractional variation, which stemmed from the amplitude variations and the baseline shifts of respiratory motion. Hence, a reasonable ITV to PTV margin and the dosimetric variation of target and organ at risks (OARs) should be evaluated for liver SBRT patients who underwent 4D CT with free-breathing.

\section{Materials and method}

\subsection{Patients}

From June 2019 to November 2019, a total of 24 patients (20 primary liver cancer patients and 4 liver metastases patients. 20 males and 4 females. The median age was 56 years old, range: $44-82$ years) were treated with SBRT under free- breathing. All the patients signed a consent form before radiotherapy. The volumetric modulated arc therapy (VMAT) technique was used for all patients $(800 \mathrm{cGy} \times 5$ fractions for 12 patients, $700 \mathrm{cGy} \times 5$ fractions for 5 patients, $900 \mathrm{cGy} \times 5$ fractions for 2 patients, $1000 \mathrm{cGy} \times 5$ fractions for 5 patients). The median dose received was $4000 \mathrm{cGy}$, the patient's treatment time was Monday, Wednesday, and Friday every week, and the total treatment time was $1-3$ weeks.

\subsection{Positioning, treatment planning, treatment}

The patients were immobilized with a thermoplastic full body mask, which was used between the xiphoid process and the navel to obtain the patient's respiratory motion signal. All patients underwent a 4D CT scan with free-breathing using a sixty-four slice spiral CT simulation (Definition AS, Siemens, Germany), with a $3 \mathrm{~mm}$ thickness. All patients were scanned with magnetic resonance imaging (MRI) for CT image fusion to determine gross tumour volume (GTV). GTV was delineated at $20 \%$ phase firstly, then the GTV contour was mapped to other nine phases, and amended manually by the oncologist. The ITV was obtained from all the ten phases. A uniform $5 \mathrm{~mm}$ ITV to PTV margin $[5,14]$ was expanded around the ITV (the original plan). The average intensity projections (AIP) from 4D CT were adopted for treatment planning and image registration with CBCT images [14,18] (XVI Release 5.0.2, Elekta Versa HD, Stockholm, Sweden). Full arc or partial arc VMAT plans were designed using the $6 \mathrm{MV}$ (1400 $\mathrm{MU} / \mathrm{min})$ or $10 \mathrm{MV}$ (2400 MU/min) photon (Flattening Filter-Free) FFF mode (Elekta Versa HD, Stockholm, Sweden).

\subsection{Acquisition of $C B C T$ images}

The first CBCT scanning was performed following the patient setup to correct liver inter-fractional errors ( $\mathrm{CBCT}_{\text {Pre-treatment }}$ ). In our study, all patients had not received implantation of fiducial markers or stent. The diaphragm $[19,20]$ and the liver shape [11] were registration manually between the CBCT and AIP to obtain liver position errors (represent the target position errors) (See Fig. 1). The investigated errors contained the three translational directions (the LR, SI and AP directions), and the rotational errors were ignored since the 6-dimensional (6D) treatment couch was not used. Move the couch to correct the inter-fractional errors if the errors were greater than or equal to $2 \mathrm{~mm}$ in any direction. The second CBCT scanning was performed and registered again to verify the liver position ( $\mathrm{CBCT}_{\text {Post-correction }}$ ). If needed (if the errors were more than $2 \mathrm{~mm}$ in any direction), the couch would be moved again to correct the errors. After liver position verification, dose delivery was executed; the intra-fractional CBCT scanning (the third CBCT scanning) was obtained simultaneously for offline analysis (XVI Release 5.0.2, Elekta Versa HD, Stockholm, Sweden). (Scanning parameters: $120 \mathrm{kV}, 40 \mathrm{~mA}, 40 \mathrm{~ms}$, 5.5 frames/second; scanning angle range from $181^{\circ}$ to $180^{\circ}$ (clockwise direction) or $180^{\circ}$ to $181^{\circ}$ (counter-clockwise).

\subsection{Match methods and errors measurements}

The baseline shifts were defined as the difference of the average tumor position (or average liver position) among all treatment fractions and the 4D CT. Our study used the diaphragm and liver shape to evaluate average tumor position between CBCT and AIP from the 4D CT. The vertebral bone registration results represented the setup errors. Then, the inter-fractional and intra-fractional liver baseline shifts were calculated as follows:

Liver baseline shifts inter $=$ Liver position $_{\text {inter }}-$ Setup $_{\text {inter }}$

Liver baseline shifts intra $=\left(\right.$ Liver $_{\text {position }}$ intra - Setup $\left._{\text {intra }}\right)-$ Liver position $_{\text {inter }}$

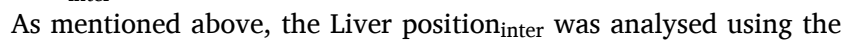
CBCT $_{\text {Pre-treatment }}$ to registration with the AIP from the 4D CT $[19,11]$ (See Fig. 1 of $j-1)$.The Setup inter represented the inter-fractional setup errors evaluated offline using vertebral bone registration between the $\mathrm{CBCT}_{\text {Pre- }}$ treatment and AIP [12] (See Fig. 1 of m-o). The same way was used to obtain the Liver position $_{\text {intra }}$ and the Setup intra between the intrafractional CBCT (the third CBCT scanning) and AIP. The correlation between the setup errors and the liver position errors were analysed.

\subsection{Amplitude variation of respiratory (diaphragm) motion}

The amplitude of diaphragm motions were compared for all treatment fractions against the AIP from the 4DCT. As for 4D CT, the motion amplitudes were measured using the MIM Maestro ${ }^{\circledR}$ system (Version 6.8.3, MIM Software Inc. Cleveland, USA). For the treatment fractions, the motion amplitudes were evaluated based on the $2 \mathrm{D}$ projections of the inter- and intra-fractional CBCT in the SI direction using a single breathing cycle (randomly extracted the projections when the $\mathrm{kV}$ X-ray source was close to the front of the patient).

Two therapists with ten years of experience performed all the 3D image registrations and measurements, and a radiotherapy oncologist with 20 years of experience reviewed these images to ensure the consistency of image registrations and measurements.2.6 ITV to PTV calculation and dosimetric variation analysis

All data was processed and analysed using SPSS 20 and EXCEL, and the correlations were analysed using Pearson correlation analysis. The ITV to PTV margin was recalculated according to the formula: PTV margin $=2.5 \Sigma+0.7 \sigma[21]$, where $\Sigma$ represent the systematic errors, which are the standard deviation (SD) of the means per patient; $\sigma$ 


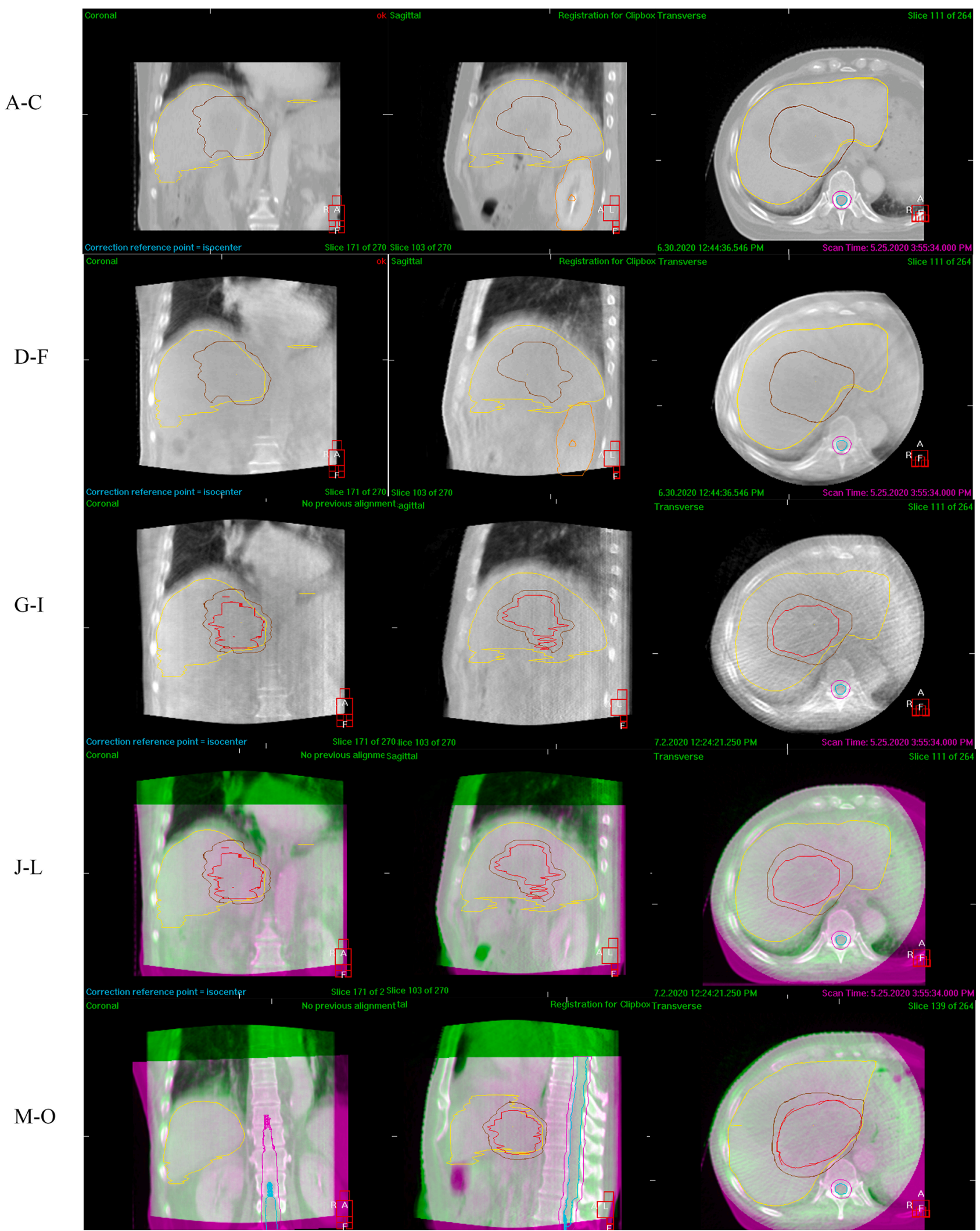

Fig. 1. Liver position errors registration and setup errors registration in the intra-fraction CBCT. (a)-(c) refer to AIP from the planning 4D CT; (d)-(f) refer to the interfractional CBCT; (g)-(i) refer to the intra-fractional CBCT; (j)-(l) refer to the liver position errors registration and (m)-(o) refer to setup errors registration in the intrafractional CBCT. 
represents the random errors, which are the root mean square of the SD's of all patients. According to the recalculated ITV to PTV margin, the robust plan was re-designed to compare the dosimetric variation using the same dose prescription of target and constrain of OARs (the paired samples T-test was used).

\section{Result}

A total of 103 paired inter- and intra-fractional CBCTs were obtained for 24 patients. The liver position errors, setup errors and liver position baseline shifts of the inter-fraction were shown in Table 1 . The systematic and random errors of the interfraction liver position errors in the LR, SI, and AP were 1.71 and $1.85 \mathrm{~mm}, 8.93$ and $5.72 \mathrm{~mm}$, and 1.29 and $1.86 \mathrm{~mm}$, respectively. The maximum setup errors stemming from vertebral bone registration and liver position baseline shifts were in the SI direction, and the corresponding ITV to PTV margins were $24.37 \mathrm{~mm}$ and $18.50 \mathrm{~mm}$. We observed that the inter-fractional liver position errors were closely related to the setup errors. The correlation coefficients were $0.80,0.70$, and 0.625 in the LR, SI and AP directions, respectively (details see Table 2).

After the $\mathrm{CBCT}_{\text {Post-correction, the intra-fractional liver position errors }}$ were significantly reduced. The systematic and random errors of the intra-fractional liver position errors in the LR, SI, and AP directions were 1.12 and $1.35 \mathrm{~mm}, 1.81$ and $2.91 \mathrm{~mm}$, and 0.94 and $1.32 \mathrm{~mm}$, respectively, with the corresponding ITV to PTV margin of $3.75 \mathrm{~mm}, 6.56 \mathrm{~mm}$, and $3.28 \mathrm{~mm}$ (Table 3 ). Because the couch correction was based on the liver position errors instead of the setup errors, there were still large residual setup errors. The maximum residual setup error was in the SI direction, with a systematic error of $5.87 \mathrm{~mm}$ and a random error of $3.83 \mathrm{~mm}$. However, the correlation between the intra-fractional liver position errors and intra-fractional setup errors were low (as reported in Table 2). The systematic and random errors of the intra-fractional liver position baseline shifts in the SI direction were 2.03 and $2.46 \mathrm{~mm}$, respectively. The corresponding ITV to PTV margin was $4.0 \mathrm{~mm}$ in LR direction, $7.0 \mathrm{~mm}$ in SI direction and $4.0 \mathrm{~mm}$ in AP direction (take these values as integers) (see Table 3).

The average diaphragm motion in $4 \mathrm{D}$ CT, the inter-fractional CBCT and the intra-fractional CBCT was $13.39 \mathrm{~mm}, 13.12 \mathrm{~mm}$ and $12.09 \mathrm{~mm}$, respectively. However, the amplitude changes of diaphragm motion for the same patient were considerable. The difference of the diaphragm motion amplitude in 4D CT and inter-fractional CBCT (103 fractions) was $0.28 \pm 5.41 \mathrm{~mm}$, with 37 of 103 fractions (36\%) exceeding $5 \mathrm{~mm}$. The difference of the diaphragm motion amplitude in 4D CT and in the intra-fractional CBCT (103 fractions) was $1.03 \pm 4.35 \mathrm{~mm}$, with $31 \%$ of fractions exceeding $5 \mathrm{~mm}$ (See Fig. $2 \mathrm{~A}$ ). Neither in the inter-fraction nor the intra-fraction, there was no significant relationship between the diaphragm motion amplitude and the liver position errors in the LR and AP directions. There was a weak correlation (the correlation coefficient was -0.4 for inter-fraction and 0.2 for intra-fraction) only in the SI direction.

The new plan was designed according to the new PTV based on the calculated ITV to PTV margin ( $4 \mathrm{~mm}$ in LR and AP directions, $7 \mathrm{~mm}$ in SI direction). Table 4 shows that the PTV volume of the new plan was significantly different from the original plan. Compared to the original
Table 2

Correlation between liver position errors and setup errors.

\begin{tabular}{|c|c|c|c|c|c|c|c|}
\hline \multirow[t]{2}{*}{ Setup errors } & & \multicolumn{3}{|c|}{$\begin{array}{l}\text { Inter-fractional liver } \\
\text { position errors }\end{array}$} & \multicolumn{3}{|c|}{$\begin{array}{l}\text { Intra-fractional liver } \\
\text { position errors }\end{array}$} \\
\hline & & LR & SI & $\mathrm{AP}$ & LR & SI & AP \\
\hline Inter- & LR & $0.80^{*}$ & -0.13 & -0.13 & & & \\
\hline fractional & SI & -0.08 & $0.70^{*}$ & -0.33 & & & \\
\hline Setup errors & AP & 0.12 & -0.14 & $\begin{array}{l}0.625 \\
*\end{array}$ & & & \\
\hline Intra- & LR & & & & $0.46^{*}$ & 0.22 & 0.07 \\
\hline fractional & SI & & & & 0.05 & $0.25^{*}$ & -0.10 \\
\hline Setup errors & AP & & & & 0.10 & -0.26 & 0.10 \\
\hline
\end{tabular}

* Correlation is significant at the 0.01 level (2-tailed).

plan, the relative PTV volume of the new plan changed from $-4 \% \sim 5.3 \%$ (Fig. 2 B). The D98, D95, Average, D50, D2 of ITV and PTV had no significant difference. To achieve the same dose coverage of ITV and PTV, the $\mathrm{D}_{\text {mean }}, \mathrm{V} 50, \mathrm{~V} 40, \mathrm{~V} 30, \mathrm{~V} 25$ of normal liver and maximum dose of the duodenum were significantly different (Table 4) (the mean value and SD were $1042.87 / 342.68 \mathrm{cGy}, 732.00 / 351.745 \mathrm{cc}, 968.80 / 441.40$ cc, $1245.93 / 521.57$ cc, $1438.47 / 560.41$ cc, $2019.77 / 1221.38$ cGy respectively for the new plan, with increased mean value was $26.7 \mathrm{cGy}$, $6.7 \%, 4.0 \%, 4.7 \%, 2.3 \%$, and $179.0 \mathrm{cGy}$ respectively).

\section{Discussion}

Chan $M$ reported [9] that there was no significant difference in registration accuracy between $3 \mathrm{D}$ CBCT and $4 \mathrm{D} \mathrm{CBCT}$ in liver radiotherapy. To reduce the CBCT scanning time, we used 3D CBCT image guidance in liver radiotherapy. Markus Oechsner reported [18] that AIP was more suitable for 3D CBCT than MIP. Bedos L et al. also reported that [14] CBCT was an average image and that registration with the planned AIP image can represent the patient's breathing pattern, so we used AIP as the reference image for registration with 3D CBCT images for liver SBRT.

Our results showed that the inter-fractional liver position errors were considerable, and there was a significant correlation between the setup errors and the liver position errors. Meanwhile, the liver position baseline shifts demonstrated significant systematic and random errors in the LR, SI and AP directions, which were $1.25 \mathrm{~mm}$ and $1.64 \mathrm{~mm}, 6.12$ $\mathrm{mm}$ and $4.57 \mathrm{~mm}$, and $1.41 \mathrm{~mm}$ and $1.79 \mathrm{~mm}$, respectively. Dhont $\mathrm{J}$ reported [10] that the liver baseline shifts between inter-fractions were $1.60 \pm 1.30,3.0 \pm 1.20 \mathrm{~mm}$, and $2.10 \pm 1.40 \mathrm{~mm}$ in the LR, SI, and AP directions, respectively. Case RB et al. [11] found that the systematic and random errors of the liver position baseline shifts in the LR, SI and AP directions were $1.50 \mathrm{~mm} / 1.80 \mathrm{~mm}, 3.10 / 3.60 \mathrm{~mm}$, and $1.60 / 2.70$ $\mathrm{mm}$, respectively for patients with free-breathing. These errors in the SI direction were significantly smaller than ours in the report mentioned above.

In our study, the liver position errors and baseline shifts in the intrafraction were significantly reduced compared to those in the interfraction, indicating that it was effective by using CBCT to correct the liver position. The baseline shifts in the LR, SI, and AP directions were $0.99 / 1.60 \mathrm{~mm}, 2.03 / 2.46 \mathrm{~mm}, 1.02 / 2.07 \mathrm{~mm}$, respectively, similar to

Table 1

Inter-fractional errors and the corresponding ITV to PTV margins (mm).

\begin{tabular}{|c|c|c|c|c|c|c|c|c|c|}
\hline \multirow[t]{2}{*}{ Inter-fractional errors } & \multicolumn{3}{|c|}{ Liver position inter-fractional errors } & \multicolumn{3}{|c|}{ Setup errors } & \multicolumn{3}{|c|}{ Liver position inter-fractional baseline shifts } \\
\hline & LR & SI & AP & LR & SI & AP & LR & SI & $\mathrm{AP}$ \\
\hline$\Sigma$ & 1.71 & 8.93 & 1.29 & 2.39 & 8.28 & 1.89 & 1.25 & 6.12 & 1.41 \\
\hline$\sigma$ & 1.85 & 5.72 & 1.86 & 2.21 & 5.24 & 1.92 & 1.64 & 4.57 & 1.79 \\
\hline PTV & 5.57 & 26.31 & 4.54 & 7.53 & 24.37 & 6.07 & 4.27 & 18.50 & 4.79 \\
\hline
\end{tabular}

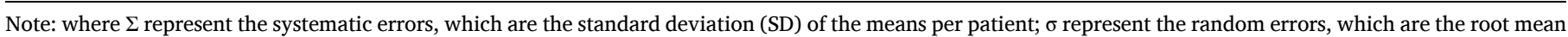
square of the SD's of all patients.

ๆ PTV margin $=2.5 \Sigma+0.7 \sigma$. 
Table 3

Intra-fractional errors and the corresponding ITV to PTV margins (mm).

\begin{tabular}{|c|c|c|c|c|c|c|c|c|c|}
\hline \multirow[t]{2}{*}{ Intra-fractional errors } & \multicolumn{3}{|c|}{ Liver position errors } & \multicolumn{3}{|c|}{ Setup errors } & \multicolumn{3}{|c|}{ Liver position baseline shifts } \\
\hline & LR & SI & $\mathrm{AP}$ & LR & SI & $\mathrm{AP}$ & LR & SI & AP \\
\hline$\Sigma$ & 1.12 & 1.81 & 0.94 & 1.21 & 5.87 & 1.28 & 0.99 & 2.03 & 1.02 \\
\hline$\sigma$ & 1.35 & 2.91 & 1.32 & 1.68 & 3.83 & 1.58 & 1.60 & 2.46 & 2.07 \\
\hline PTV & $3.75(4.0)^{*}$ & $6.56(7.0)^{※}$ & $3.28(4.0)^{*}$ & 4.20 & 17.35 & 4.31 & $3.61(4.0)^{*}$ & $6.80(7.0)^{*}$ & 4.00 \\
\hline
\end{tabular}

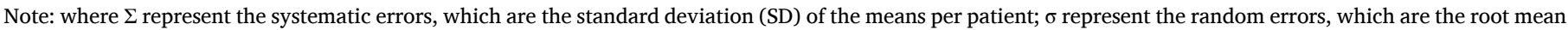
square of the SD's of all patients.

I PTV margin $=2.5 \Sigma+0.7 \sigma$.

* These values in parentheses are integers.

A

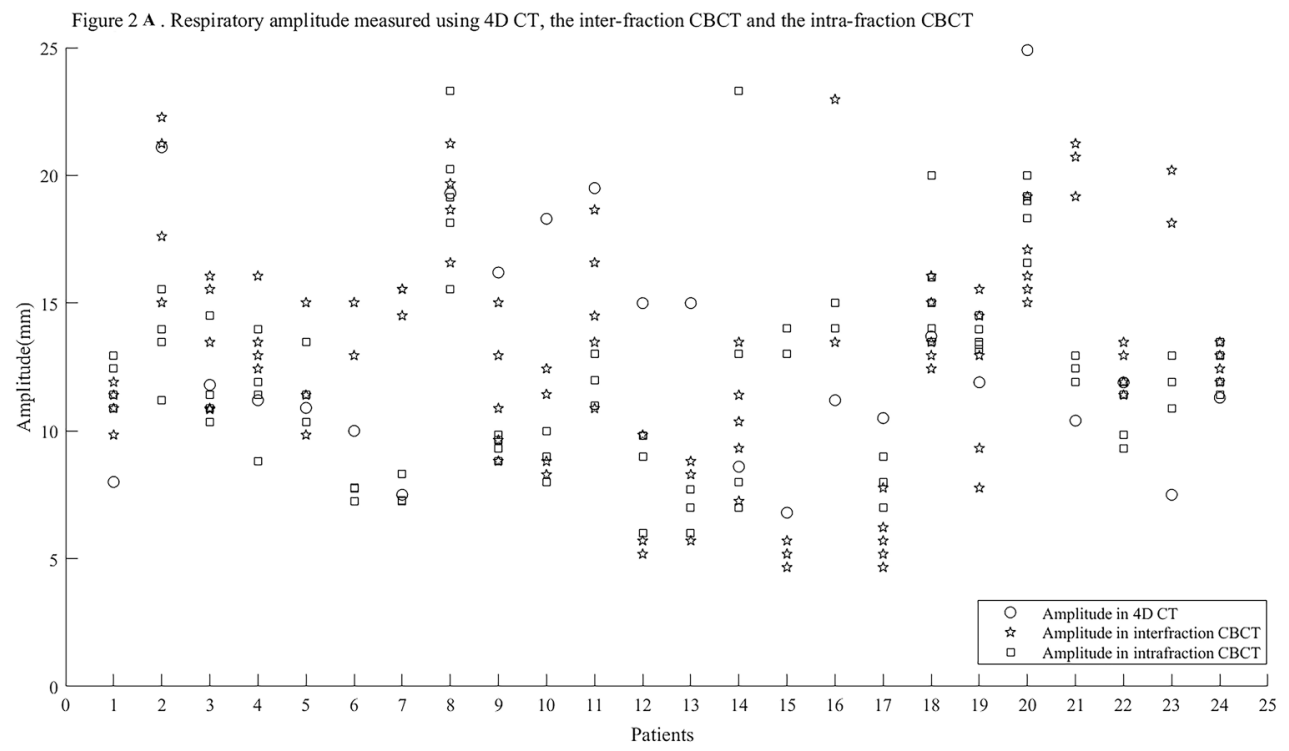

B

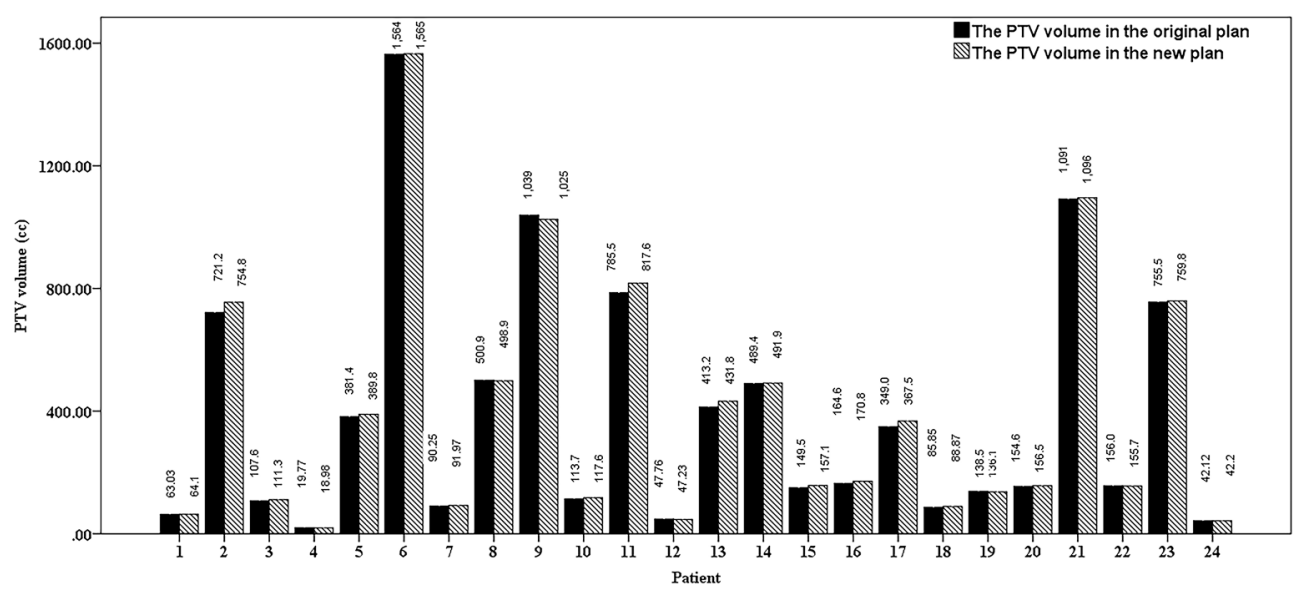

Fig. 2. Respiratory amplitude changes and the comparison of PTV volume. A. Respiratory amplitude measured using 4D CT, the inter-fractional CBCT and the intrafractional CBCT; B. Comparison of PTV volume between the original and new plans.

the report by Case RB et al. $(1.20 / 2.20 \mathrm{~mm} 1.40 / 3.00 \mathrm{~mm} 1.00 / 1.90$ $\mathrm{mm}$, respectively) [11] and Poulsen PR et al $(0.9 \mathrm{~mm}, 1.4 \mathrm{~mm}, 0.8 \mathrm{~mm})$ [17]. Bedos L. et al. [14] used a $\mathrm{kV}$ planar imaging to acquire the endexpiratory image to analyse the patient's intra-fractional errors and found that the $99 \%$ intra-fractional errors in the SI direction were within $5 \mathrm{~mm}$, which were smaller than in our study. However, the images were collected before the treatment dose delivery, and the acquisition time was limited, which cannot fully represent intra-fractional errors.
Another report showed that the medians (ranges) of the baseline shifts were $1.87(0.06-12.04) \mathrm{mm}, 0.35(0-3.39) \mathrm{mm}$ and $1(0.02-7.21) \mathrm{mm}$ in the SI, LR and AP directions, respectively [22]. Our study used the intrafractional $\mathrm{CBCT}$ to evaluate the intra-fraction baseline shifts compared to the baseline in AIP. The average frame number of the intra-fractional CBCT was 467 frames, and the average acquisition time was $84.9 \mathrm{~s}$, including approximately 20 breathing cycles. Xu Q et al. [23] used liver markers to evaluate the baseline displacement, and they found that the 
Table 4

Paired samples T-test between the original and the new plans.

\begin{tabular}{lllll}
\hline \multicolumn{2}{l}{ Dosimetric parameter } & $\begin{array}{l}\text { The original plan } \\
\text { (Mean/SD) }\end{array}$ & $\begin{array}{l}\text { The new plan } \\
\text { (Mean/SD) }\end{array}$ & $P_{\text {value }}$ \\
\hline PTV volume & & $407.91 / 409.56$ & $413.68 / 411.56$ & 0.017 \\
ITV Dose & D98 & $4145.40 / 755.44$ & $4145.07 / 750.12$ & 0.975 \\
& D95 & $4338.33 / 700.24$ & $4338.47 / 690.77$ & 0.991 \\
& average & $4732.33 / 697.89$ & $4717.20 / 690.14$ & 0.327 \\
& D50 & $4731.53 / 692.87$ & $4717.33 / 683.98$ & 0.425 \\
PTV Dose & D2 & $5228.87 / 826.24$ & $5189.60 / 814.48$ & 0.251 \\
& D98 & $3585.07 / 801.78$ & $3565.27 / 802.48$ & 0.072 \\
& D95 & $3849.07 / 750.00$ & $3841.67 / 753.33$ & 0.108 \\
& average & $4563.53 / 687.98$ & $4562.87 / 685.94$ & 0.954 \\
& D50 & $4605.07 / 681.67$ & $4609.60 / 675.67$ & 0.764 \\
Normal & D2 & $5190.00 / 813.29$ & $5164.27 / 807.87$ & 0.343 \\
& $\mathrm{D}_{\operatorname{mean}}$ & $1016.07 / 342.66$ & $1042.87 / 342.68$ & 0.001 \\
& V50 & $696.40 / 355.81$ & $732.00 / 351.745$ & 0.018 \\
& V40 & 944.73 .436 .58 & $968.80 / 441.40$ & 0.021 \\
& V30 & $1223.53 / 527.39$ & $1245.93 / 521.57$ & 0.028 \\
& V25 & 1414.13 .560 .29 & $1438.47 / 560.41$ & 0.035 \\
& V23 & $1501.00 / 581.26$ & $1449.27 / 682.96$ & 0.535 \\
& $\mathrm{D}_{\max }$ & $1812.54 / 1211.23$ & $2019.77 / 1221.38$ & 0.048 \\
Duodenum & $\mathrm{D}_{\max }$ & $2362.42 / 551.74$ & $2384.75 / 531.13$ & 0.573 \\
\hline
\end{tabular}

The original plan used a uniform $5 \mathrm{~mm}$ PTV margin around the ITV. The new plan used a PTV margin of $4 \mathrm{~mm}$ in LR and AP directions, $7 \mathrm{~mm}$ in SI direction around the ITV.

baseline displacement of LR, AP and SI were $2.10 \pm 2.30 \mathrm{~mm}, 2.90 \pm$ $2.80 \mathrm{~mm}$, and $6.40 \pm 5.50 \mathrm{~mm}$, respectively. According to these reports and our findings, the baseline shifts were significant during treatment. it means that an additional ITV to PTV margin is needed to compensate the baseline shifts occurring at treatment.

Dhont $\mathrm{J}$ et al. [10] used a single metal marker to analyse the respiratory motion in the SI direction. The amplitude changes over $5.00 \mathrm{~mm}$ were $53 \%$ for the inter-fraction and $28 \%$ for the intra-fraction. Shimohigashi Y S et al. [24] reported that the average movement in the SI direction was $5.30 \mathrm{~mm}$, and the maximum value was $14.80 \mathrm{~mm}$ using abdominal pressure. The medians (ranges) of the intra-fractional amplitude variation across all patients were $4.30(1.60-6.00) \mathrm{mm}, 0.5$ $(0.20-2.20) \mathrm{mm}$ and $1.50(0.30-3.30) \mathrm{mm}$ in the SI, LR and AP directions, respectively [22]. In the current study, the variation of the respiratory motion amplitude among 4D CT, inter-fractional CBCT and intra-fractional CBCT in the SI direction was small (the average amplitude was $13.39 \mathrm{~mm}, 13.12 \mathrm{~mm}$ and $12.09 \mathrm{~mm}$, respectively), indicating that the variation of overall patients' respiratory motion amplitude was relatively small. However, for individual patients' respiratory motion, there was significant variation during the whole treatment processing. Compared to the amplitude measured in 4D CT, the rate of amplitude difference greater than $5 \mathrm{~mm}$ was $31 \%$ of cases. Poulsen PR et al. [17] reported that the tumor motion range was considerably larger during treatment delivery (mean: $17.3 \mathrm{~mm}$; range: $7.3-35.7 \mathrm{~mm}$ ) than in the planning 4DCT scan (mean: $7.6 \mathrm{~mm}$; range: $4.0-14.7 \mathrm{~mm}$ ) showing that the 4DCT scans failed to capture the range of anatomy changes occurring at treatment. According to these findings, we recommended using two image facilities (such as the fluoroscopy image, 4D CT, or 4D CBCT) and more than one time to evaluate the motion amplitude and baseline position before treatment planning.

Considering the baseline shifts, Dhont $\mathrm{J}$ et al. recommended the 8 mm ITV to PTV margin for the liver [10]. Worm ES et al. [25] reported that when the target area was delineated using 4D CT with moderate ventilation, a $10 \mathrm{~mm}$ ITV to PTV margin in the SI direction and $5 \mathrm{~mm}$ margins in AP and LR directions should be used. The baseline shifts represent systematic errors, which introduced a systematic dose deviation, and the amplitude changes were random errors, which blurred the dose around the target edge [21]. The reduction of CTV $D_{95}$ was in mean $6.0 \%$ for single fractions and $5.3 \%$ for treatment courses due to the intrafractional errors [17]. According to the results in our study, the ITV to PTV margin should be $4 \mathrm{~mm}$ in LR and AP directions, and $7 \mathrm{~mm}$ in the SI direction. Using the recalculated ITV to PTV margin, we found that the relative PTV volume of the new plan changed from $-4 \% \sim 5.3 \%$. To achieve the same dose coverage of PTV, the $D_{\text {mean }}$ V50, V40, V30, V25 of normal liver and maximum dose of the duodenum were significantly different (Table 4). However, these dosimetric parameters would be decreased if we used a stricter constrained objective function of OARs. Even so, all these dosimetric parameters of normal tissue are clinically acceptable. Therefore, the new ITV to PTV margin is a clinically acceptable method to ensure the target's dose coverage if there is no effective strategy to reduce intra-fractional errors.

There is a limitation in our study. Since metal markers, stent or lipiodol were not implanted in or near the tumour, the 3D deviation of tumour position cannot be analysed using $2 \mathrm{D}$ projections. Therefore, implanted metal markers [26], lipiodol[27], 4D CBCT[9,27]or MR [4] could be used to obtain more target information and to further analyse the amplitude changes and baseline shifts of respiratory motion during dose delivery.

\section{Conclusions}

Significant amplitude changes and baseline shifts of respiratory motion occurred during dose delivery compared with those in 4D CT. Using the ITV to PTV margin of $4.0 \mathrm{~mm}$ (LR), $7.0 \mathrm{~mm}$ (SI), and $4.0 \mathrm{~mm}$ (AP) can compensate the intra-fractional baseline drifts to ensure the target dose coverage, and keep the dose constrain of normal tissues at an acceptable level.

\section{Authors' contributions}

Lu Zeng collected data and drafted the manuscript. Xin Wang designed the patients' treatment planning and reviewed the image registration. Jidan Zhou, Pan Gong, Xuetao Wang, Xiaohong Wu, Zhonghua Deng, Bin Li and Denghong Liu collected and analyzed the data. Renming Zhong designed the study, revised and finally approved the manuscript. All authors read and confirmed the manuscript.

\section{Funding}

This work was supported by the 1.3.5 Project for Disciplines of Excellence, West China Hospital, Sichuan University (ZYJC18008), the 1.3.5 project for disciplines of excellence-Clinical Research Incubation Project, West China Hospital, Sichuan University (No: 2021HXFH029) and the Science and Technology Support Program of Sichuan province, China (No.2021JDKP0070).

\section{Ethics}

This study was approved by the Ethics Committee of West China School of Medicine, Sichuan University (No.20191128).

\section{Declaration of Competing Interest}

The authors declare that they have no known competing financial interests or personal relationships that could have appeared to influence the work reported in this paper.

\section{Acknowledgment}

This work was supported by the Science and Technology Support Program of Sichuan province, China (No. 2021YFQ0065, 2021JDKP0070), the 1.3.5 Project for Disciplines of Excellence, West China Hospital, Sichuan University (ZYJC18008), and the 1.3.5 project for disciplines of excellence-Clinical Research Incubation Project, West China Hospital, Sichuan University (No: 2021HXFH029). 


\section{References}

[1] Zhong R, Wang J, Jiang X, He Y, Zhang H, Chen N, et al. Hypofraction radiotherapy of liver tumor using cone beam computed tomography guidance combined with active breath control by long breath-holding. Radiother Oncol 2012;104(3): 379-85.

[2] Yang M, Timmerman R. Stereotactic ablative radiotherapy uncertainties: delineation. Setup and Motion Semin Radiat Oncol 2018;28(3):207-17.

[3] Li W-Z, Liang Z-W, Cao Yi, Cao T-T, Quan H, Yang Z-Y, et al. Estimating intrafraction tumor motion during fiducial-based liver stereotactic radiotherapy via an iterative closest point (ICP) algorithm. Radiat Oncol 2019;14(1). https://doi. org/10.1186/s13014-019-1401-2.

[4] Rosenberg SA, Henke LE, Shaverdian N, Mittauer K, Wojcieszynski AP, Hullett CR, et al. A multi-institutional experience of MR-guided liver stereotactic body radiation therapy. Adv Radiat Oncol 2019;4(1):142-9.

[5] Gargett M, Haddad C, Kneebone A, Booth JT, Hardcastle N. Clinical impact of removing respiratory motion during liver SABR. Radiat Oncol 2019;14:93.

[6] Poulsen PR, Murtaza G, Worm ES, Ravkilde T, O’Brien R, Grau C, et al. Simulated multileaf collimator tracking for stereotactic liver radiotherapy guided by kilovoltage intrafraction monitoring: Dosimetric gain and target overdose trends. Radiother Oncol 2020;144:93-100.

[7] Borm KJ, Oechsner M, Wiegandt M, Hofmeister A, Combs SE, Duma MN. Moving targets in 4D-CTs versus MIP and AIP: comparison of patients data to phantom data. BMC Cancer 2018;18:760.

[8] Bradley JD, Nofal AN, El Naqa IM, Lu W, Liu J, Hubenschmidt J, et al. Comparison of helical, maximum intensity projection (MIP), and averaged intensity (AI) 4D CT imaging for stereotactic body radiation therapy (SBRT) planning in lung cancer. Radiother Oncol 2006;81(3):264-8.

[9] Chan M, Chiang CL, Lee V, Cheung S, Leung R, Wong M, et al. Target localization of $3 \mathrm{D}$ versus $4 \mathrm{D}$ cone beam computed tomography in lipiodol-guided stereotactic radiotherapy of hepatocellular carcinomas. PLOS ONE 2017;12(4):e0174929.

[10] Dhont J, Vandemeulebroucke J, Burghelea M, Poels K, Depuydt T, Van Den Begin R, et al. The long- and short-term variability of breathing induced tumor motion in lung and liver over the course of a radiotherapy treatment. Radiother Oncol 2018;126(2):339-46.

[11] Case RB, Sonke J-J, Moseley DJ, Kim J, Brock KK, Dawson LA. Inter- and intrafraction variability in liver position in non-breath-hold stereotactic body radiotherapy. Int J Radiat Oncol Biol Phys 2009;75(1):302-8.

[12] Zhang T, Wang W, Li Y, Jin J, Wang S, Song Y, et al. Inter- and intrafractional setup errors and baseline shifts of fiducial markers in patients with liver tumors receiving free-breathing postoperative radiation analyzed by cone-beam computed tomography. J Appl Clin Med Phys 2014;15(6):138-46.

[13] Case RB, Moseley DJ, Sonke JJ, Eccles CL, Dinniwell RE, Kim J, et al. Interfraction and intrafraction changes in amplitude of breathing motion in stereotactic liver radiotherapy. Int J Radiat Oncol Biol Phys 2010;77(3):918-25.

[14] Bedos L, Riou O, Aillères N, Braccini A, Molinier J, Moscardo CL, et al. Evaluation of reproducibility of tumor repositioning during multiple breathing cycles for liver stereotactic body radiotherapy treatment. Rep Pract Oncol Radiother 2017;22(2): $132-40$.

[15] Nankali S, Worm ES, Hansen R, Weber B, Høyer M, Zirak A, et al. Geometric and dosimetric comparison of four intrafraction motion adaptation strategies for stereotactic liver radiotherapy. Phys Med Biol 2018;63(14):145010. https://doi. org/10.1088/1361-6560/aacdda.

[16] Li R, Han B, Meng B, Maxim PG, Xing L, Koong AC, et al. Clinical implementation of intrafraction cone beam computed tomography imaging during lung tumor stereotactic ablative radiation therapy. Int J Radiat Oncol Biol Phys 2013;87(5): 917-23.

[17] Poulsen PR, Worm ES, Petersen JBB, Grau C, Fledelius W, Høyer M. Kilovoltage intrafraction motion monitoring and target dose reconstruction for stereotactic volumetric modulated arc therapy of tumors in the liver. Radiother Oncol 2014; 111(3):424-30.

[18] Oechsner M, Chizzali B, Devecka M, Combs SE, Wilkens JJ, Duma MN. Registration uncertainties between 3D cone beam computed tomography and different reference CT datasets in lung stereotactic body radiation therapy. Radiat Oncol 2016;11:142.

[19] Yang J, Cai J, Wang H, Chang Z, Czito BG, Bashir MR, et al. Is diaphragm motion a good surrogate for liver tumor motion? Int J Radiat Oncol Biol Phys 2014;90(4): 952-8.

[20] Kawahara D, Ozawa S, Kimura T, Nakashima T, Aita M, Tsuda S, et al. Availability of applying diaphragm matching with the breath-holding technique in stereotactic body radiation therapy for liver tumors. Phys Med 2016;32(4):557-61.

[21] Vanherk M. Errors and margins in radiotherapy. Semin Radiat Oncol 2004;14(1): 52-64.

[22] Liang Z, Liu H, Xue J, Hu B, Zhu B, Li Q, et al. Evaluation of the intra- and interfractional tumor motion and variability by fiducial-based real-time tracking in liver stereotactic body radiation therapy. J Appl Clin Med Phys 2018;19(3): 94-100.

[23] Xu Q, Hanna G, Grimm J, Kubicek G, Pahlajani N, Asbell S, et al. Quantifying rigid and nonrigid motion of liver tumors during stereotactic body radiation therapy. Int J Radiat Oncol Biol Phys 2014;90(1):94-101.

[24] Shimohigashi Y, Toya R, Saito T, Ikeda O, Maruyama M, Yonemura K, et al. Tumor motion changes in stereotactic body radiotherapy for liver tumors: an evaluation based on four-dimensional cone-beam computed tomography and fiducial markers. Radiat Oncol 2017;12(1). https://doi.org/10.1186/s13014-017-0799-7.

[25] Worm ES, Høyer M, Fledelius W, Poulsen PR. Three-dimensional, time-resolved, intrafraction motion monitoring throughout stereotactic liver radiation therapy on a conventional linear accelerator. Int J Radiat Oncol Biol Phys 2013;86(1):190-7.

[26] Bertholet J, Worm ES, Fledelius W, Høyer M, Poulsen PR. Time-resolved intrafraction target translations and rotations during stereotactic liver radiation therapy: Implications for marker-based localization accuracy. Int J Radiat Oncol Biol Phys 2016;95(2):802-9.

[27] Chan MKH, Lee V, Chiang CL, Lee FAS, Law G, Sin NY, et al. Lipiodol versus diaphragm in 4D-CBCT-guided stereotactic radiotherapy of hepatocellular carcinomas. Strahlenther Onkol 2016;192(2):92-101. 